\title{
CORPUS Corpus
}

Archivos virtuales de la alteridad americana

Vol 4, No 2 2014

Julio / Diciembre 2014

\section{L'image du sauvage dans le théâtre quechua et l'iconographie des queros (Pérou, XVII-XVIII)}

La imagen del salvaje en el teatro quechua y en la iconografía de los queros

(Perú, XVII-XVIII)

The figure of the savage in quechua theatre and in queros iconography

\section{Rossella Martin}

\section{OpenEdition}

\section{Journals}

Electronic version

URL: http://journals.openedition.org/corpusarchivos/1236

DOI: $10.4000 /$ corpusarchivos. 1236

ISSN: 1853-8037

\section{Publisher}

Diego Escolar

\section{Electronic reference}

Rossella Martin, « L'image du sauvage dans le théâtre quechua et l'iconographie des queros (Pérou, XVII-XVIII) », Corpus [En línea], Vol 4, No 2 | 2014, Publicado el 19 diciembre 2014, consultado el 30 abril 2019. URL : http://journals.openedition.org/corpusarchivos/1236 ; DOI : 10.4000/ corpusarchivos.1236

This text was automatically generated on 30 April 2019. 


\section{L'image du sauvage dans le théâtre quechua et l'iconographie des queros (Pérou, XVII-XVIII)}

La imagen del salvaje en el teatro quechua y en la iconografía de los queros

(Perú, XVII-XVIII)

The figure of the savage in quechua theatre and in queros iconography

Rossella Martin

\section{EDITOR'S NOTE}

Fecha de recepción del original: 05/09/2014

Fecha de aceptación para publicación: 24/10/2014

\section{Introduction}

1 Bien que les échanges de biens et de populations entre les trois principaux écosystèmes du Pérou, la costa, la sierra et la selva, aient été très intenses depuis au moins le deuxième millénaire avant J.-C. (Renard-Casevitz et Saignes, 1986, p. 22), les contreforts amazoniens des Andes restaient, à l'époque coloniale, une région partiellement inexplorée. Les Incas l'avaient appelée Antisuyu ou « secteur des Antis », ${ }^{1}$ désignant par métonymie sous le nom d'Antis les diverses ethnies qui vivaient sur ce territoire. Les rapports entre les Incas et les peuples de la selva semblent avoir été assez fluctuants: les alliances politiques et matrimoniales qui assuraient aux deux partenaires l'accès à des ressources et à des services diversifiés, étaient généralement suivies par des ruptures qui entraînaient des razzias d'un côté, des expéditions punitives et des tentatives d'annexion de l'autre. ${ }^{2}$ C'est au tout début du règne de l'Inca Thupa Yupanki (dernier quart du XV siècle), que les chroniqueurs ayant recueilli les témoignages des khipukamayuq, ${ }^{3}$ situent la plus 
importante expédition dirigée contre les peuples de la selva. Cette expédition semble avoir tourné court à cause du climat insalubre et des maladies qui affaiblirent l'armée inca, déjà éprouvée par une guerre d'attaques surprises à laquelle elle n'était pas habituée :

Fama es entre los orejones del Cuzco que Topainga Yupangue atravesó con grande ejército esta montaña, y que fueron muy difíciles de conquistar [...] Bien adentro de estas montañas y espesuras afirman que hay gente tan rústica que no tienen casa ni ropa, antes [sic] como animales, matando con flechas aves y bestias las que pueden para comer [...] (Cieza de León, [1553] 1984 : vol. I, p. 120).

On raconte - mais Cieza reconnait ne pas l'avoir vu de ses propres yeux - que "por tentación del demonio " les Antis s'accouplent avec de gros singes en engendrant des créatures monstrueuses. D'ailleurs, il est impossible de les considérer comme des hommes à part entière car « no tienen habla, sino un gemido o aullido temeroso $»^{4}$ (Cieza de León, [1553] 1984 : vol. I, p. 120).

C'est pourtant aux Antis que Manqu Inka demanda aide et protection en 1536, lorsqu'il réussit à échapper au contrôle des occupants espagnols et organisa le siège de Cuzco. Son fils, le chroniqueur T'itu Kusi Yupanki, raconte que les flèches enflammées des Antis incendièrent les toits en paille de la capitale inca (Tito Cusi Yupanqui, [1570] 1973, p. 92). Obligé de replier ses troupes le long du fleuve Vilcanota, Manqu demeura quelque temps à Ollantaytambo avant de rejoindre Vitcos, dans la cordillère de Vilcabamba. C'est là, en territoire anti, que lui et ses successeurs donnèrent vie à un nouvel État inca qui sut tenir tête au gouvernement colonial jusqu'en 1572.

4 Les expéditions militaires espagnoles ainsi que les tentatives missionnaires d'implantation dans les basses terres s'étant heurtées aux mêmes difficultés qui avaient autrefois freiné l'expansionnisme inca: cinquante ans après le témoignage de Cieza de León l'image de l'Anti restait inchangée. Les peuples de la forêt continuaient à être perçus comme des êtres mi-homme mi-animal. Huamán Poma de Ayala (1615) les appelle anti runa mikhuq « les Antis anthropophages» (Guaman Poma de Ayala, [1615] 2005 : t. I, pp. 242-43) et Garcilaso de la Vega (1609) n’a pour eux que des mots de mépris :

Los que viven en los Antis comen carne humana, son más fieros que tigres, no tienen dios ni ley, ni saben qué cosa es virtud ; tampoco tienen ídolos ni semejanza de ellos ; adoran al demonio cuando se les representa en figura de algún animal o de alguna serpiente y les habla. (Garcilaso de la Vega, [1609] 1976 : vol. I, p. 29).

Dans les chroniques coloniales, les peuples de la forêt sont également appelés ch'unchu, nom qui a remplacé de nos jours l'ethnonyme Anti d'origine précolombienne et qui sert à désigner de façon dépréciative aussi bien les natifs de la selva que les gens incultes incapables de vivre en société. L'étymologie de ce terme demeure obscure, à moins qu'on ne tente un rapprochement - comme le propose César Itier - avec des mots quechuas commençant par la racine chu- et faisant référence à un être animé ou inanimé qui a perdu sa vigueur ou sa consistance à cause, par exemple, de l'action combinée du soleil et du gel, comme chullpa "(ancêtre) racorni » et ch'uñu "pomme de terre déshydratée " (Itier, 2004, pp. 21-22). Ce qui permettrait de mettre en relation nos ch'unchu avec le mythe des Hintil ou ancêtres " gentils ", rapporté par la tradition orale. Ces Hintil auraient vécu à une époque où la terre n'était éclairée que par la lune et où aucune loi, sinon celle du plus fort, ne régissait la vie sociale. La plupart des Hintil seraient morts brûlés par le premier lever du soleil, identifié à Jésus-Christ. Mais certains d'entre eux se seraient sauvés en suivant à la nage le cours des rivières jusqu'à la yunka, ${ }^{5}$ où l'épaisse végétation les aurait protégés du qarwa ruphay « la chaleur dorée $» .^{6}$ Les ch'unchu seraient donc les 
descendants de ces ancêtres païens, dont ils auraient perpétué les mœurs en choisissant de vivre dans un état de sauvagerie anarchique (Renard-Casevitz et Saignes, 1986, p. 41).

Ni le dictionnaire anonyme de 1586 ni celui de Diego González Holguín de 1608 - pour une fois trop concis - ne donnent d'informations sur l'origine du mot ch'unchu. Dans le premier, la classe de référents à laquelle l'entrée renvoie, ce sont les Indiens eux-mêmes : «Chunchu. Yndios de guerra, de la Cordillera » (Anónimo, [1586] 1951: art. chunchu) ; dans le second, c'est le territoire: "Chhunchu. Vna prouincia o de Andes de guerra » (Holguín [1608] 1989: art. chhunchu). Les deux définitions partagent cependant un attribut essentiel, la guerre, à laquelle les ch'unchu semblent définitivement être associés.

On peut, de ce fait, partir d'un premier constat: les traits distinctifs qui caractérisent l'image de l'habitant de la selva dans le discours colonial sont sa sauvagerie et sa bellicosité. Or, partant du principe que toute image est un fait de culture et le vecteur d'une certaine idéologie, il faut se demander ce que l'image coloniale de l'Anti peut nous révéler de la société qui l'a élaborée. Une société qui, de toute évidence, définissait sa propre identité par rapport à la représentation d'une altérité conçue comme inférieure et qui se servait de ce langage symbolique pour transmettre ses propres valeurs.

Dans cet objectif, nous examinerons deux corpus appartenant à la même période historique, le XVII ${ }^{e}$ et le XVIII ${ }^{e}$ siècle, et à la même aire géographique et culturelle, les hautes terres andines de la vice-royauté du Pérou. Il s'agit, d'une part, des drames du théâtre quechua colonial et, d'autre part, des représentations d'Antis peintes sur les gobelets en bois appelés queros. À ma connaissance, leur mise en parallèle n'a jamais été effectuée sinon d'une manière à peine ébauchée par Verena Liebscher, qui a essayé d'établir une filiation thématique entre Ollantay et la scène figurée sur un quero conservé au Museum für Völkerkunde de Munich (Liebscher, 1986, p. 61).

9 Les manuscrits des drames quechuas, ${ }^{7}$ dont sont tirés les extraits présentés dans cet article, ont été réunis par César Itier, qui est en train d'en donner la transcription paléographique, la translittération en graphie quechua moderne et la traduction en langue espagnole dans la base de données créée par le groupe Langues Générales d'Amérique du Sud (LANGAS). C'est à ce site web que je renvoie le lecteur, ne pouvant pas donner les textes en intégralité dans l'espace offert par la revue: http:// josephine.inalco.perso.sfr.fr/recherche_lexicale.php

10 Pour ce qui concerne le corpus des queros coloniaux avec des représentations d'Antis, j'ai pu étudier les exemplaires conservés au Musée du quai Branly de Paris et au Museo de América de Madrid, ${ }^{8}$ grâce à une bourse d'études postdoctorale obtenue en 2008 . Le caractère répétitif et codifié de l'iconographie des queros coloniaux m'autorise à porter mon analyse sur un nombre très limité de pièces, choisies parmi les plus représentatives de chaque type de scène.

\section{Le corpus du théâtre quechua colonial}

11 Le théâtre quechua colonial naît et se développe à Cuzco au cours des XVII ${ }^{\mathrm{e}}$ et XVIII ${ }^{\mathrm{e}}$ siècles. Six pièces nous sont parvenues. Les plus anciennes sont deux autos sacramentales écrits vers le milieu du XVII ${ }^{\mathrm{e}}$ siècle par Juan de Espinosa Medrano : El robo de Proserpina y sueño de Endimión et $E l$ hijo pródigo. Suivent trois comedias marianas, ${ }^{9} \mathrm{El}$ pobre más rico de Gabriel Centeno de Osma (fin du XVII ${ }^{\mathrm{e}}$ ou début du XVIII ${ }^{\mathrm{e}}$ siècle) et les anonymes Uska Pawqar et El milagro del Rosario, qui dateraient du milieu du XVIII ${ }^{e}$ siècle. Nous avons enfin 
la comedia d'argument pseudo-historique Ollantay, composée en 1782 par Antonio Valdez.

12 À l'exception peut-être de El milagro del Rosario, œuvre qui est moins bien écrite et qui intègre de nombreux éléments de la tradition orale, ces pièces montrent une excellente maîtrise des codes littéraires du théâtre baroque espagnol: versification poly-métrique avec prédominance des octosyllabes et maniement de la rime (bien qu'avec de nombreuses irrégularités) ; division en trois actes avec insertion de fragments lyriques et présence de personnages-types comme le galán et le gracioso dans les comedias; action renfermée en un seul acte, présence de personnages allégoriques et thématique eucharistique dans les autos sacramentales. Il s'agit de toute évidence d'une production savante, destinée à un public urbain formé à la culture occidentale. Aussi ne doit-on pas considérer le fait que nos pièces sont écrites dans une langue indigène comme un indice révélateur de l'origine ethnique de leurs auteurs. Grâce à sa diffusion importante en tant que langue de communication inter-ethnique au sein de l'empire inca, le quechua avait été choisi par le Troisième Concile de Lima (1582-83) pour servir les besoins de l'évangélisation. Un abondant corpus, comprenant des manuels de catéchèse, des grammaires et des dictionnaires, avait rapidement vu le jour. Le quechua était ainsi devenu la lengua general la plus diffusée de la vice-royauté et, dans la ville de Cuzco - dont la population était à dominante indienne -, toutes les composantes ethniques de la société le comprenaient et le parlaient avec fluidité. La langue de nos drames est par ailleurs un idiome élaboré, fruit d'un projet cultivé et puriste (Lienhard, 1992, p. 161), riche en archaïsmes lexicaux - mots sortis de l'usage - et en archaïsmes sémantiques mots utilisés en un sens sorti de l'usage. Espinosa Medrano semble avoir été un métis de mère indienne (Vitulli, 2011, pp. 13-15) ; on ignore l'origine ethnique de Gabriel Centeno de Osma (Meneses, 1983, p. 378) ; quant à Antonio Valdez, ses biographes hésitent entre l'identité métisse et créole (Meneses, 1977 ; 1983 ; Porras, 1954a et b ; 1955). Tous les trois étaient des ecclésiastiques, comme ce fut probablement le cas pour les auteurs de l'Usca Paucar et El milagro del Rosario. Dans le Cuzco colonial, où l'Église détenait le monopole de la culture et où l'éducation de l'élite autochtone était confiée aux Jésuites, les religieux formaient une classe d'intellectuels bilingues, étroitement liée aux groupes seigneuriaux indigènes.

\section{L'image de l'habitant de la selva dans le théâtre quechua colonial}

Le théâtre quechua colonial étant à visée édifiante et moralisatrice, les protagonistes de nos drames sont tous des pécheurs qui finissent par se racheter et expier leurs fautes. Dans les autos sacramentales d'Espinosa Medrano, la figure du diable tentateur - Pluton dans El robo de Proserpina et Mundo dans El hijo pródigo - évoque clairement l'image du souverain inca, dont il affiche les insignes du pouvoir et, notamment, la maskapaycha. ${ }^{11}$ Dans les comedias marianas du XVIII siècle, en revanche, tout en conservant certains attributs de l'ancien seigneur du Tawantinsuyu, le démon s'incarne sous les traits de l'habitant de la yunka.

14 C'est ainsi qu'en essayant de mettre en garde son maître contre Nina Kiru, la « Dent de Feu », le gracioso Qispillu lui fait immédiatement remarquer l'apparence hybride hommeanimal du mystérieux étranger : 


\begin{tabular}{|l|l|}
\hline Qispillu & Quespillo \\
Yawrit'itu, awkilláy, & Yawrit'itu, mon cher seigneur, \\
haku aykipullasun kaymanta & fuyons vite d'ici! \\
Ima runapunich kayqa? & Quel genre d'homme est-il? \\
Yaqullan ancha kuyuptinmi & Quand sa cape se soulève \\
sikinñiqta qhawarini. & j'entrevois ses fesses! \\
Ima manchaytam rikuni : & Quelle monstruosité que j'aperçois?! \\
k'usilluhina chupasapam kasqa, & Comme le singe, il a une longue queue! \\
imamanpas raykukullachwantaq. & Qu'il ne nous arrive pas quelque malheur! \\
(El pobre más rico, vv. 722-730) & . \\
\hline
\end{tabular}

La comparaison de l'étranger à un singe pour souligner son aspect démoniaque est classique : le diable est traditionnellement désigné comme « le singe de Dieu » car il essaie d'en imiter les actions. Mais le singe est également un animal qui vit dans la forêt et son association avec le démon sert à introduire l'habitat de ce dernier. C'est en effet dans la selva que Nina Kiru cherche à entraîner le naïf protagoniste de El pobre más rico. Et lorsque celui-ci lui demande où il va l'emmener, Qispillu intervient en disant: Yunkamanchari kuka pallaq, «Mais dans la yunka pour recueillir la coca!» (El pobre más rico, v. 802). On comprend mieux la boutade du gracioso si l'on se souvient qu'à l'époque coloniale, la coca - dont les plantations fleurissaient sur les versants amazoniens des Andes - était appelée l'« herbe du diable» par les autorités religieuses, qui l'associaient aux rites païens préhispaniques. Dans les trois comedias marianas, dont l'action se passe peu de temps après la Conquête, le fait que le noble indigène qui en est le protagoniste se laisse convaincre de suivre le démon dans la selva, symbolise le risque encouru par les populations autochtones de retomber dans le paganisme. Les Indiens sont considérés par l'Église comme des Chrétiens encore chancelants et, par conséquent, des proies faciles pour Satan. Et à une époque où l'adjectif « christianisé » est synonyme de « civilisé », il est naturel que le diable de nos drames habite le territoire le plus sauvage et le plus dangereusement « exotique » de la vice-royauté, autrement dit la yunka.

Son appartenance à ce milieu lourd de menaces pour les hommes des hautes terres andines, le démon de l'Uska Pawqar la révèle déjà dans son nom : Yunka Nina, le « Feu de la forêt ». La description qu'il fait de son riche royaume, où la pauvreté n'existe pas et où l'or et l'argent coulent à flots (Uska Pawqar, vv. 530-561), rappelle inévitablement à l'esprit l'image du Paititi. C'est dans ce légendaire eldorado amazonien, version mythifiée du dernier repaire de Manqu Inka, que des trésors inestimables auraient été cachés aux conquérants espagnols. Tellement bien cachés, d'ailleurs, que toute expédition organisée dans le but de les retrouver, s'était soldée par un cuisant échec. Mais à l'instar du pays de cocagne de la tradition européenne, le pays de l'abondance païen décrit par Yunka Nina, est une terre où les lois naturelles et les valeurs morales sont perverties à l'extrême et où l'homme finit par s'égarer dans la vaine recherche du plaisir, comme les protagonistes de nos drames l'apprennent à leurs dépens.

D'argument profane et situé au temps des anciens Incas, Ollantay semble de prime abord ne rien avoir en commun avec les drames évoqués jusqu'ici. Cependant, l'histoire de la rébellion du général de l'Antisuyu qui, après avoir défié le pouvoir de Cuzco pendant dix 
ans, est pardonné et réintégré dans ses fonctions par l'Inca Thupa Yupanki, peut être interprétée comme une nouvelle version de l'histoire de la chute et du rachat de l'homme. Excepté que, cette fois, la figure de l'Inca ne possède aucune connotation diabolique ; au contraire, le portrait de Thupa Yupanki - le même Thupa Yupanki qui, d'après les chroniques, aurait mené une importante expédition en territoire anti - se conforme au modèle littéraire du bon prince chrétien (Martin 2011, pp. 123-146). Face à lui, Ollantay n'apparaît que plus coupable et sa révolte passible d'être condamnée. Car tout en ayant reçu une éducation inca, celui qui a été élevé au rang de général par son seigneur, refuse de se soumettre à l'autorité de celui-ci lorsqu'elle va à l'encontre de ses projets. ${ }^{12}$ Sa véritable nature reprend le dessus et, ayant tourné le dos à la ville de Cuzco, Ollantay revendique son identité de ch'unchu devant ses armées d'Antis : [...] kasaq qamkunawan ch'unchu (Justiniani, v. 795), " uni à vous, je serai un ch'unchu ». Puissant et orgueilleux, ce personnage s'apparente aux diverses incarnations de Lucifer qui, dans les autos sacramentales et dans les comedias marianas, racontent leur combat contre Dieu à la tête des légions des anges rebelles ${ }^{13}$ :

\begin{tabular}{|l|l|}
\hline Ullantay & \\
Hunu hunu waranqata & Ollantay \\
Antikunata llullaspa & Mille milliers \\
Trompeurs & d'Antis, en les persuadant avec des mots \\
suyuykunata tuqllaspa & j'emmènerai comme un bouclier. \\
Saqsawamanpim rikunki & Tu verras à Sacsayhuaman \\
runayta phuyutahina, & mes hommes comme des nuages, \\
chaypim sayarinqa nina, \\
yawarpim chaypi puñunki. \\
là le feu se lèvera,
\end{tabular}

18 Mais à la différence de Lucifer, Ollantay est seulement un homme qui, en dépit de ses faiblesses, finit par demander humblement pardon à son Inca : Ama tapuwaychu, Yaya, / huchaykum tukuypi phuqchin (Justiniani, vv. 1587-1588), « Ne me questionne pas, Père, / partout nos crimes débordent».

Pour comprendre les changements qui sont intervenus dans la caractérisation de la figure du Diable qui, dans le passage des autos sacramentales du XVII e siècle aux comedias du XVIII e, a troqué l'habit inca pour celui de ch'unchu, il faut considérer que nos comedias ont été écrites en pleine «Renaissance inca ». Depuis le texte fondateur de John H. Rowe (1957), on sait combien la diffusion au Pérou de la deuxième édition des Comentarios reales, parue à Madrid en 1723, fut importante dans le processus de reconstruction identitaire de l'élite indigène. L'image idéalisée de la société inca véhiculée par Garcilaso, rencontra un énorme succès auprès de la noblesse indienne qui, à partir de la fin du XVII ${ }^{e}$ siècle et tout au long du XVIII ${ }^{e}$, se fixa comme objectif la réhabilitation de la mémoire de ses ancêtres. À Cuzco, les descendants des anciens seigneurs du Tawantinsuyu défilaient dans les cérémonies publiques avec des vêtements et des emblèmes incas; ils se transmettaient des légendes relatives à la geste épique de leurs ancêtres - légendes dont est probablement issu Ollantay - et décoraient leurs maisons d'objets traditionnels comme les queros. On peut dès lors supposer que les queros coloniaux n'ont pas revêtu une simple 
valeur symbolique mais qu'ils ont aussi rempli une fonction de complément visuel à la tradition orale.

\section{Les Antis peints sur les queros coloniaux}

D'origine précolombienne, les queros sont de hauts gobelets à libations en forme généralement tronconique, dite également de « cloche inversée » (Liebscher, 1986, p. 20). Le mot quero vient du quechua qiru et signifie « bois "; à l'époque des Incas, il existait une catégorie d'artisans, les qirukamayuq («les responsables du bois»), spécialisés dans la fabrication de vases et d'autres objets en bois destinés à la noblesse. La tradition des queros survécut à la Conquête et connut une véritable floraison au cours des XVII ${ }^{\mathrm{e}}$ et XVIII e siècles. Les qirukamayuq coloniaux satisfaisaient à la demande en symboles incaïques de l'élite indigène, des rangs de laquelle ils étaient issus. Les principaux centres de production s'établirent à proximité des villes andines les plus densément peuplées, comme Ayacucho, Cuzco et Potosí, tandis que de nouvelles formes (coupe, calice, tête anthropomorphe et zoomorphe) firent leur apparition à côté de la forme tronconique traditionnelle. En même temps, la surface des vases se recouvrit de motifs figuratifs (hommes, fleurs et animaux), réalisés avec une laque à base de pigments d'origine végétale et minérale. ${ }^{14}$ Le répertoire iconographique était très codifié et comprenait seulement une douzaine de sujets, ${ }^{15}$ parmi lesquels figurent des scènes complexes ayant pour protagonistes les habitants de la selva.

21 À l'intérieur de ce groupe, on peut distinguer les scènes d'inspiration incaïque de celles d'inspiration coloniale. Par leur continuité thématique, les premières forment une sorte de « séquence narrative » qui se déroule en trois temps :

1. les Incas et les Antis s'affrontent sur le champ de bataille

2. les Antis prisonniers sont conduits enchaînés à la cour de l'Inca

3. les Antis «domestiqués» soutiennent le parasol impérial de l'Inca ou de la Coya (« reine »)

Quant aux scènes d'inspiration coloniale, elles montrent exclusivement des défilés de ch'unchu qui sont en train de danser ou de jouer d'un instrument de musique d'origine occidentale.

Que symbolise donc cet ennemi que les Incas combattent dans les scènes de type 1 mais qui finit par être accueilli au sein du monde civilisé, comme le témoignent les scènes de type 2 et 3 ainsi que les défilés de ch'unchu dans les villes coloniales? 


\section{Civilisés versus barbares}

\section{conflit armé entre deux groupes de guerriers vus de profil.}

IMAGE N 1 : Dessin de la scène développée sur le quero $n^{\circ} 7511$ du Museo de América (Archives du MA)

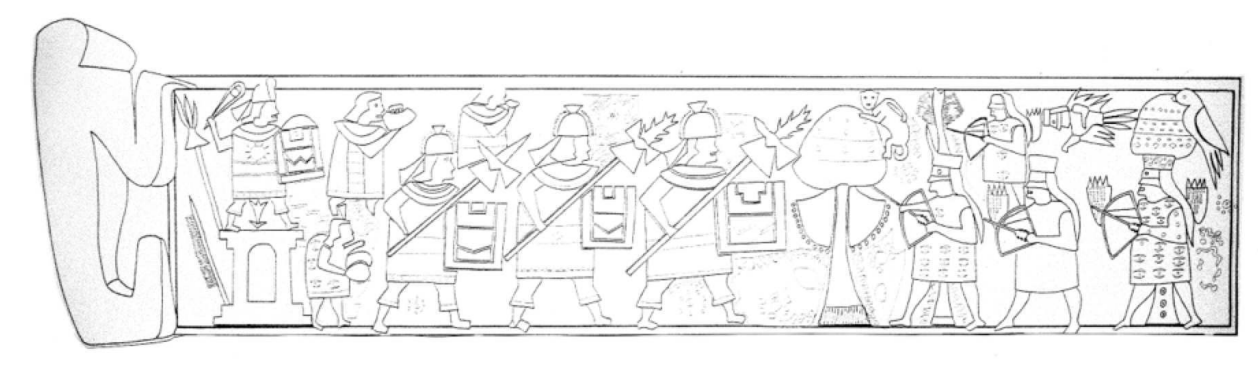
The considère le cadre spatial et le système référentiel des personnages, on constat prime abord que le groupe de gauche, constitué de quatre guerriers incas et de trois musiciens, occupe les deux tiers de la scène. Cette "supériorité iconographique » n'est qu'en apparence numérique car le groupe de droite est lui aussi formé de sept figures, dont quatre guerriers antis, un perroquet, un singe et une sorte d'oiseau anthropomorphe à tête d'Anti. Si le groupe de gauche " envahit » la scène donnant l'impression d'empiéter sur le territoire de ses ennemis, c'est surtout à cause de la taille des guerriers incas - plus imposante que celle des Antis - et des dimensions de leurs armes. Trois d'entre eux, en effet, portent un bouclier rectangulaire (pullqanqa) et une longue hallebarde (champi). Ils sont coiffés d'un casque (chuku) qui accroît leur stature. Le quatrième, armé lui aussi d'un bouclier et d'une fronde (warak'a), est situé sur une forteresse (pukara). Face à eux, les Antis disposent seulement d'un petit arc (p'iqta) et d'un étui à flèches (wach'i churana). Comme les Incas, trois guerriers antis sont rangés en file indienne dans une posture qui suggère le mouvement. Mais comme ils occupent un espace réduit par rapport à leurs adversaires - espace que la présence de trois arbres de chunta rétrécit davantage -, on a l'impression qu'ils sont en train de reculer devant l'avancée des Incas.

L'attention du spectateur est attirée par d'autres éléments qui mettent en évidence l'aspect exotique des Antis : les guerriers ont le visage tatoué, les cheveux longs et sont habillés avec une tunique sans manches parsemée d' « yeux » comme la peau maculée du jaguar. Leur apparence mi-homme mi-bête est renforcée par l'association avec deux animaux de la jungle, le singe et le perroquet, et avec l'être anthropozoomorphe volant qui représente vraisemblablement un chaman. ${ }^{16}$ Par contraste, les Incas ont les cheveux coupés courts et portent une tunique (unku) décorée de tuqapu ${ }^{17}$ et des pantalons anachronisme colonial dont la fonction est certainement celle de refléter le haut degré de civilisation atteint par les Incas. Le quatrième guerrier dirige les opérations du haut de la forteresse, en faisant tournoyer une fronde. Sa position et le disque solaire qu'il affiche sur la poitrine - emblème de la royauté inca d'invention coloniale - indiquent que c'est lui qui donne les ordres. Les guerriers sont accompagnés par trois musiciens qui jouent du tambour (wankar) et de la conque marine (pututu), instruments que les Incas utilisaient à la guerre. 
out oppose les Incas civilisés - dont la structure architectonique, les tuniques décorées de toqapu et l'organisation hiérarchique sont les symboles - et les Antis. Par le paysage qui leur sert de décor - palmiers et fruits de la jungle -, par les animaux qui les accompagnent - singes et perroquets -, par les tatouages et les peaux de jaguar dont ils sont revêtus, ces derniers se définissent immédiatement comme des figures de l'altérité, étrangères au groupe social et ethnique qui produit et utilise les queros, c'est-à-dire l'élite indigène urbaine. Deux peuples, deux espaces géographiques, deux types de société s'affrontent sur le champ de bataille. En s'identifiant à leurs ancêtres incas, les peintres des queros donnent une représentation de l'infériorité physique et culturelle des Antis qui laisse deviner leur infériorité morale.

\section{Le miroir des Incas}

Suivant cette clé de lecture, la séquence narrative constituée par les scènes de type 1, 2 et 3 , peut facilement s'interpréter comme une illustration de cet idéal civilisateur que Garcilaso prête aux anciens seigneurs du Tawantinsuyu et que le mouvement de la Renaissance inca exalte.

IMAGE $\mathrm{N}^{\circ} 2$ : Dessin de la scène développée sur le quero $\mathrm{n}^{\circ} 71.1946 .7 .200 \mathrm{D}$ du Musée du quai Branly

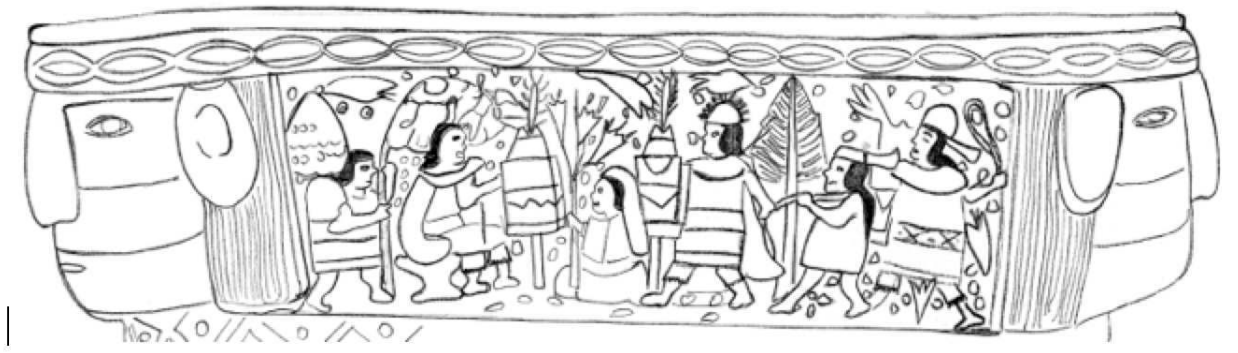

Poursuivons notre analyse, et portons à présent notre attention sur une scène à plusieurs personnages, représentée dans la partie postérieure d'un vase "Anti-céphalomorphe ». De gauche à droite, un bossu (k'umillu) soutient le parasol impérial (achiwa) au-dessus de la tête de l'Inca, assis sur une tiyana; une Coya agenouillée lui offre des fleurs de chiwanway, tandis qu'un guerrier inca conduit un prisonnier anti de taille plus petite, les mains attachées et la corde au cou. Un deuxième guerrier inca les suit en faisant tournoyer sa fronde. Or, si l'on se reporte aux témoignages laissés par les chroniqueurs, les Incas emmenaient à Cuzco les chefs des ennemis vaincus pour qu'ils soient foulés aux pieds du souverain avant qu'on ne leur coupe la tête. Il en va autrement dans les Comentarios reales, où les empereurs incas, à l'instar du Thupa Yupanki d'ollantay, sont toujours prêts à pardonner à leurs ennemis et à les faire profiter de la pax incaica. Les scènes de type 3 montrent clairement que c'est cette vision idéalisée de la monarchie inca que les peintres des queros veulent véhiculer. En comparant les deux dessins ci-dessous, nous pouvons en effet constater qu'ils reproduisent la même scène, à une importante différence près. 


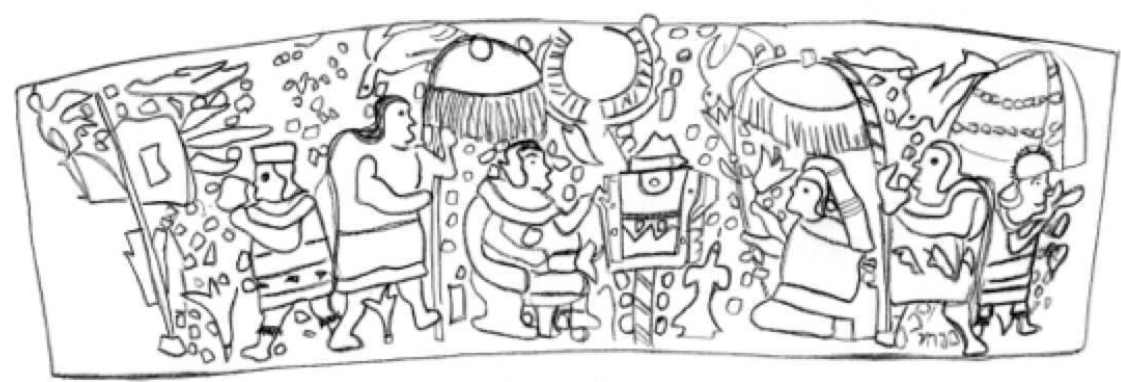

IMAGE $N^{\circ} 4$ : Dessin de la scène développée sur le quero $n^{\circ} 7502$ du Museo de América

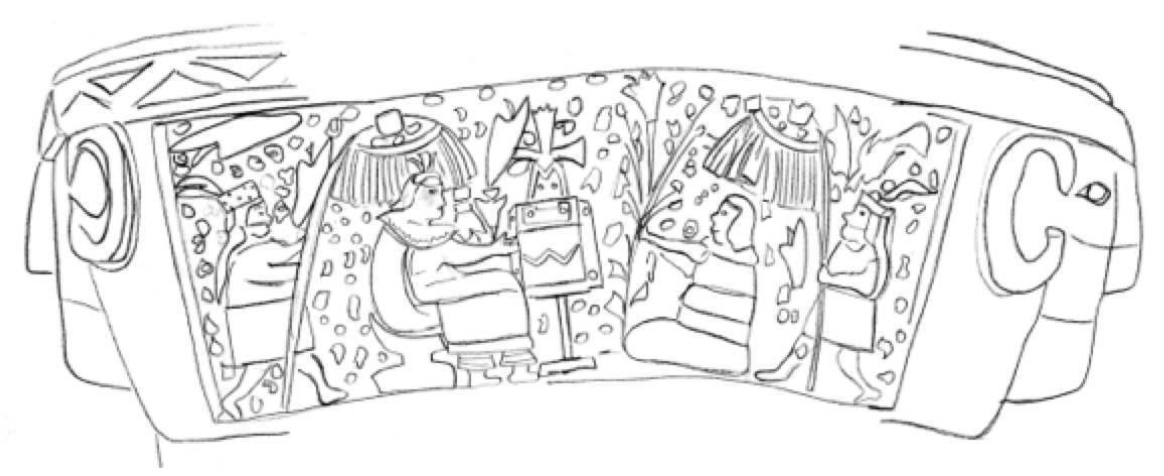

Dans les deux cas, les personnages au centre de la composition sont un Inca assis sur une tiyana et une Coya agenouillée en face de lui. Derrière chaque personnage, un serviteur soutient un parasol de plumes. Mais, alors que dans la première scène la fonction de serviteur est remplie par le bossu - figure traditionnellement associée à la vie de cour-, ${ }^{18}$ dans la deuxième les serviteurs sont deux personnages au visage peint et coiffés de plumes. Il s'agit visiblement d'Antis qui, après avoir été capturés et emmenés à Cuzco, ont été intégrés à la société inca.

Il est probable que notre séquence narrative raconte, tout particulièrement, l'histoire de la conquête de l'espace par les ancêtres fondateurs de la dynastie inca, Manqu Qhapaq et Mama Uqllu. D'après le mythe narré par Garcilaso, en effet, s'étant rendu compte que les hommes vivaient dans un état misérable, le Soleil aurait envoyé sur la terre ses deux fils pour qu'ils leur enseignent les bienfaits de la vie policée (Garcilaso de la Vega, [1609] 1976 : t. I, pp. 36-42). Les sauvages représentés sur les queros symboliseraient donc les populations primitives du Pérou, que le couple d'ancêtres mythiques allait transformer en une nouvelle humanité. De cette manière, les peintres des queros montraient que les Incas n'étaient pas des barbares, qui avaient dû attendre la venue des Espagnols pour apprendre à se conduire en êtres civilisés - ainsi que les présentait un certain courant de pensée d'origine tolédiste. ${ }^{19}$ En inversant la perspective, ils les montraient au contraire comme des civilisateurs investis de la mission d'adoucir les mœurs des peuples assujettis. Autrement dit, l'image de l'Autre permettait à l'élite indigène de se mettre en valeur : par la représentation idéalisée des anciens seigneurs du Pérou, elle participait ainsi à la 
"guerre iconographique » (Stastny, 1982) que se livraient les divers secteurs de la société coloniale. Une guerre dont l'enjeu pour l'élite indigène était - rappelons-le - la réhabilitation de la mémoire de ses ancêtres, au nom desquels elle pouvait revendiquer une participation plus active à la vie politique du pays.

Mais notre séquence narrative transmet aussi un autre message. Car si la victoire des Incas sur les Antis symbolise la victoire de la civilisation sur la barbarie, ces images montrent également que l'intégration des peuples sauvages est considérée comme nécessaire à l'instauration d'une société inca qui soit vraiment fondatrice et unificatrice.

\section{Les défilés de ch'unchu ou l'intégration possible}

Le quero $\mathrm{n}^{\circ} 7531 \mathrm{du}$ Museo de América présente, dans la bande supérieure, un cortège de huit personnages, dont six ont le visage peint et sont ornés de majestueuses couronnes de plumes.

IMAGE N 5 : Dessin de la scène développée sur le quero $n^{\circ} 7531$ du Museo de América (Archives du MA)

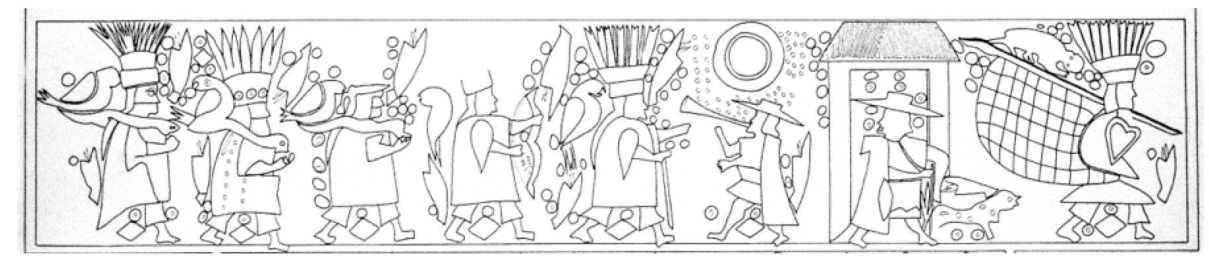

Il s'agit de danseurs ch'unchu et la scène se situe dans une ville coloniale, comme en témoigne la présence d'une maison en arrière-plan et de deux musiciens habillés à la mode espagnole et jouant d'instruments d'origine européenne : un tambour similaire à ceux qui étaient utilisés par les régiments d'infanterie et une trompette en métal. ${ }^{20}$ Le personnage qui ouvre la parade - appelé caporal dans les danses de ch'unchu actuelles porte un drapeau en damier multicolore, comme ceux qui sont connus en Bolivie sous le nom de wifala. Un singe se tient accroché à la hampe du drapeau. Derrière les deux musiciens et le petit chien qui les accompagne suivent cinq ch'unchu coiffés de plumes. Ils portent un arc ou un bâton en forme de serpent, sur lequel est juché un perroquet. À la différence des Antis dans les scènes d'inspiration incaïque, les ch'unchu coloniaux ne sont pas revêtus de peaux de bêtes mais portent des tuniques, des pantalons et des capes.

Si l'origine des bailes de chunchos pourrait remonter à l'époque préhispanique, où des délégations de l'Orient selvatique se rendaient à Cuzco pour chanter et danser devant l'Inca à l'occasion des fêtes principales (Guaman Poma de Ayala, [1615] 2005 : t. I, pp. 242-45), les sources coloniales attestent que des gens costumés en ch'unchu prenaient régulièrement part aux festivités catholiques les plus importantes. Cette tradition s'est maintenue vivante jusqu'à nos jours: parmi les groupes de comparsas qui participent annuellement au pèlerinage du Señor de Quyllu R'iti (7-13 juin) et à la fête de la Virgen del Carmen à Paucartambo (15-19 juillet), il y a les Capac chunchos. ${ }^{21}$ Ces derniers constituent le cortège d'honneur de la Vierge, sur laquelle ils veillent jalousement et pour laquelle ils dansent inlassablement (Brachetti, 2005, pp. 156-190). Une légende raconte qu'au cours d'une incursion à Paucartambo, les ch'unchu avaient attaqué l'église et criblé de flèches la statue de la Vierge. Ils l'avaient ensuite jetée dans l'Amaru Mayu («la rivière du serpent»), qui depuis lors avait pris le nom de «Madre de Dios» pour avoir sauvé la 
statue de la noyade et l'avoir déposée sur ses berges..$^{22}$ Ayant fini par se convertir au christianisme et par se repentir de leur acte, les ch'unchu étaient devenus les fidèles gardiens de la Vierge et ses danseurs préférés. D'après la légende, l'année où ils n'avaient pas pris part à la fête, la statue aurait pâlit et toute sorte de malheurs se seraient abattus sur le village (Roel Pineda, 1950, pp. 60-61).

Le récit de l'étrange aventure arrivée à la statue de la Vierge de Paucartambo ne fait que confirmer l'hypothèse qu'en représentant des Antis "apprivoisés » et des danseurs ch'unchu, les peintres des queros voulaient montrer la progressive mais inévitable intégration de ces derniers à la société civilisée. Car l'obstination dont faisaient preuve les habitants de la selva en refusant d'être englobés dans un système social hiérarchisé et centralisé, constitua un défi majeur autant pour l'empire inca que pour le gouvernement colonial. Fondés sur un pouvoir hégémonique à vocation universaliste, ni l'un ni l'autre ne pouvaient accepter qu'à l'intérieur de leur territoire il y eût des populations échappant à leur contrôle. Autrement dit, des populations qui ne pouvaient pas êtres recensées et, par conséquent, astreintes au service de la mit' ${ }^{\prime 23} \mathrm{ni}$ au paiement des tributs. Qui plus est, la présence de peuples s'adonnant librement à des rites païens et à des pratiques considérées comme immorales au sein de la très catholique vice-royauté du Pérou, était une source constante de préoccupation. Pour les autorités politiques et religieuses, il était indispensable d'assimiler ces populations qui menaçaient l'ordre constitué.

C'est probablement le sens qu'il faut donner aux mots prononcés par le grand prêtre Willaq Umu, alter ego du curé Antonio Valdez dans Ollantay, invitant l'Inca Thupa Yupanki à incorporer l'Antisuyu à son Empire :

\begin{tabular}{|l|l|}
\hline Willaq Umu Huillac Omo & \\
Huk ankatam kicharqayku & Nous avons ouvert la poitrine \\
qhasqunta qhawaykunapaq, & d'un aigle pour regarder et \\
sunqunmanta riqsinapaq: & connaître (le futur) à travers son cœur. \\
ch'usaqllatam tarirqayku. & Mais nous l'avons trouvée vide. \\
Chay ankam Antisuyuyki. & Il faut le réunir rapidement (à l'Empire), \\
q'asakapunmi chaykuna. & ses gens nous manquent. \\
Chaytam kunan watupuyki. & C'est mon augure pour toi. \\
(Justiniani, vv. 1158-1165) & \\
\hline
\end{tabular}

41 Quelques décennies après la rédaction d'ollantay, l'auteur de la version quechua de la Proclama a los habitantes de Ultramar (1813) rappelait que l'évangélisation des peuples de la selva était également une priorité pour le gouvernement représentatif de Cadix :

Ichaqa ñawpaq-ñawpaqta sunqunchista aysaqqa ch'unchukunaq hatun yunkankunapi mana Diosta riqsispa kawsayninmari. Chaypaqmari chay awki ministro Ultramar ñisqa ñawinkunata sunquntawanpas ñawpaq-ñawpaqta t'ikrarinqa chay q'ala runakunata chiqaq samiman llapata llullaspa Santo Yayakunata kachaspa tukuyta atipaspaqa Diospa ñanñinman aysamuyta Bautismopi christianota hurquspa hanaqpachaman thatkirinanpaq. (Rivet, 1951-56 : t. II, p. 256)

Mais ce qui nous préoccupe tout particulièrement, c'est que les habitants de la forêt profonde vivent sans connaître Dieu. C'est pourquoi ce noble seigneur, appelé ministre d'Outremer, tournera ses yeux et son cœur en premier lieu vers ces gens, en les 
entrainant tous vers le vrai bonheur, en leur envoyant des saints pères, en vainquant leur résistance et en faisant d'eux, à travers le baptême, des Chrétiens qui suivent le chemin de Dieu, afin qu'ils dirigent résolument leurs pas vers le Ciel.

De cette revue certainement trop rapide d'un double corpus qui regroupe des documents littéraires et iconographiques, il est tout de même possible de tirer un certain nombre de conclusions. L'image que les chroniques coloniales nous renvoient des habitants de la selva péruvienne, désignés sous les noms génériques d'Antis ou de ch'unchu, est celle que la culture occidentale conquérante nourrit de ses préjugés : hommes bestiaux, sans dieu ni loi, belliqueux et cannibales. Au XVII et au XVIII ${ }^{e}$ siècle, des représentants de l'élite indigène andine reprennent cette image à leur compte. Aussi bien les descendants des Incas que les caciques, à qui les œuvres du théâtre quechua et les queros sont destinés, veulent être considérés comme des modèles d'intégration et de conversion réussie au christianisme, des parangons de policía cristiana. C'est la conditio sine qua non pour trouver sa place dans le secteur dominant de la société coloniale. La frontière à marquer avec les "autres" Indiens doit par conséquent être bien nette: géographique, morale et culturelle. Les hommes des basses terres amazoniennes deviennent les contre-modèles négatifs de ceux des hautes terres andines, les Barbares païens face aux Chrétiens civilisés. La représentation du Sauvage comme créature démoniaque vient ainsi alimenter le portrait de Satan, brossé par les auteurs des comedias marianas. L'infériorité physique et technologique des guerriers antis guerroyant contre les Incas, est mise en évidence par les peintres des queros, qui font des uns les faire-valoir des autres. Les Antis servent à célébrer le rôle de civilisateurs que les Incas coloniaux attribuent à leurs ancêtres. C'est la raison pour laquelle les "mauvais Sauvages » finissent par se transformer en "bons Sauvages »: le ch'unchu Ollantay fait acte d'allégeance à son seigneur, les Antis policés font office de serviteurs à la cour de l'Inca. Les scènes représentant des défilés de ch'unchu dans des villes coloniales, attestent que l'intégration des Sauvages s'est bel et bien réalisée.

Sans doute la volonté de l'élite indigène de magnifier l'action civilisatrice des Incas coïncidait-elle avec la volonté des autorités espagnoles de donner une image harmonieuse et idéalisée de la société coloniale. Comme les couples mixtes qui sourient heureux avec leur enfant dans les cuadros de castas du vice-roi Amat (1770), les ch'unchu qui dansent et jouent de la musique dans les scènes peintes sur les queros, entretiennent l'image utopique d'une société régie par l'entente sereine de ses diverses composantes ethniques et culturelles. Une image qui donne certainement une représentation déformée de la réalité mais qui n'en reste pas moins « marquante ", car elle appartient à cette catégorie d'images qui qualifient le moment historique de leur élaboration autant qu'elles sont qualifiées par lui.

\section{BIBLIOGRAPHY}

Anónimo ([1586] 1951). Vocabulario y phrasis en la lengua General de los indios del Perú llamada Quichua... Lima : Edición del Instituto de Historia. Universidad de San Marcos. 


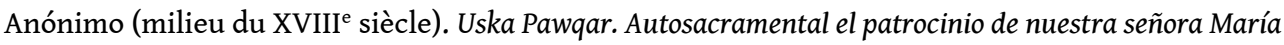
santicima en Copacavana. Disponible dans: http://josephine.inalco.perso.sfr.fr/

recherche_lexicale.php

Anónimo (milieu du XVIII ${ }^{\mathrm{e}}$ siècle). El milagro del Rosario. Disponible dans:

http://josephine.inalco.perso.sfr.fr/recherche_lexicale.php

Brachetti, Á. (2005). El año en fiestas. La convivencia con los dioses en los Andes del Perú. Madrid : Ministerio de Cultura. Museos estatales. Museo de América.

Centeno de Osma, G. (fin du XVII ${ }^{\mathrm{e}}$ - début du XVIII ${ }^{\mathrm{e}}$ siècle). El pobre más rico. Disponible dans: http://josephine.inalco.perso.sfr.fr/recherche_lexicale.php

Cieza de León, P. de ([1553] 1984). Crónica del Perú, Primera parte. En : Obras completas, vol. I. (C. Sáenz de Santa María, Ed.). Madrid : Consejo Superior de Investigaciones Cientificas/Instituto «Gonzalo Fernández de Oviedo ».

Cummins, Th. B. F. (2004). Brindis con el Inca. La abstracción andina y las imágenes coloniales de los queros. Lima : Fondo Editorial UNMSM/Universidad Mayor de San Andrés/Embajada de los Estados Unidos de América.

Espinosa Medrano, J. de ([fin de la décennie 1640] 2010). El robo de Proserpina y sueño de Endimión. Autosacramental en quechua. Edición, traducción y estudio preliminar de César Itier. Lima: Institut Français d'Études Andines (IFEA)/Pontificia Universidad Católica del Perú (PUCP).

Espinosa Medrano, J. de (fin de la décennie 1640). Autosacramental alegórico del Hijo Pródigo. Disponible dans: http://josephine.inalco.perso.sfr.fr/recherche_lexicale.php

Flores Ochoa, J. A., Kuon Arce, E., Samanez Argumedo, R. (1998). Queros. Arte inka en vasos ceremoniales. Lima: Banco de Crédito del Perú.

García-Bedoya, C. (1998). Teatro quechua colonial, barroco andino y renacimiento inca. Actas del I Encuentro Internacional de Peruanistas, 3-6 de septiembre 1996 (pp. 325-338). Lima : Universidad de Lima.

Garcilaso de la Vega, Inca ([1609] 1976). Comentarios reales de los Incas (A. Miró Quesada, Ed.). Caracas: Biblioteca Ayacucho, 2 vol.

González Holguín, D. ([1608] 1989). Vocabulario de la lengua general de todo el Perú llamada lengua qquichua o del Inca. Lima: Universidad Nacional Mayor de San Marcos.

Guaman Poma de Ayala, F. ([1615] 2005). Nueva crónica y buen gobierno. (Franklin Pease G.Y., Ed.). 3 tomes. Lima: Fondo de Cultura Ecónomica.

Gudemos, M. (2004). Canto, danza y libación en los Andes. La música en las pinturas de los 'queros' del Museo de América. Madrid: Ministerio de Cultura. Museos estatales. Museo de América.

Itier, C. (2004). La littérature orale quechua de la région de Cuzco - Pérou. Paris: Karthala/Langues O'.

Liebscher, V. (1986). La iconografía de los queros. Lima: G. Herrera Editores.

Lienhard, M. (1992). La voz y su huella. Escritura y conflicto étnico-cultural en América Latina 1492-1988. (3a ed. rev. y aum.). Lima: Horizonte.

Martin, R. (2010). Tupac Yupanqui ou le modèle du prince parfait. Étude de l'autre protagoniste d' ollantay. Bulletin de l'Institut Français d'Études Andines, 40 (1), 123-146.

Meneses, T. L. (1977). Datación y paternidad del drama "Apu Ollantay". Revista de Arte, Ciencias y Humanidades. Lima: Universidad Nacional Mayor de San Marcos (UNMSM). Fondo Editorial 17, 49-82. 
Meneses, T. L. (1983). Teatro quechua colonial. Antología. Lima: Ediciones Edubanco.

Porras Barrenechea, R. (1954a). El padre Valdez, autor del Ollantay. El Comercio. Lima, 18-11-1954. Réédité in Indagaciones peruanas. El legado quechua (Porras Barrenechea R., ed.), 390-393. Lima: Universidad Nacional Mayor de San Marcos (UNMSM). Fondo Editorial/Instituto Raúl Porras Barrenechea.

Porras Barrenechea, R. (1954b). La paternidad definitiva de Ollantay. El Comercio, Lima, 19-11-1954. Réédité in Indagaciones peruanas. El legado quechua (Porras Barrenechea R., ed.), 393-396. Lima: Universidad Nacional Mayor de San Marcos (UNMSM). Fondo Editorial/Instituto Raúl Porras Barrenechea.

Porras Barrenecha, R. (1955). El Ollantay y Antonio Valdez . El Comercio, Lima, 17-11-1955. Réédité in Indagaciones peruanas. El legado quechua. (Porras Barrenechea R., ed.), 396-399. Lima: Universidad Nacional Mayor de San Marcos (UNMSM). Fondo Editorial/Instituto Raúl Porras Barrenechea.

Proclama a los habitantes de Ultramar / Sinchikunay Mamaquchaq chimpanpi kawsaq runakunaman (1813) in Rivet, P., Créqui-Montfort G. de (1951-1956). Bibliographie des langues aymará y kichua. Paris: Institut d'Ethnologie, t. II, 247-260.

Renard-Casevitz, F-M., Saignes Th., Taylor A-C. (1986). L'Inca, l'Espagnol et les sauvages. Rapports entre les sociétés amazoniennes et andines $d u$ XVe au XVIIe siècle. Paris: Recherche sur les civilisations (ERC).

Roel Pineda, J. (1950). Música y Coreografía Tradicionales. La danza de los 'C'uncos' de Paucartambo, Tradición. Revista peruana de cultura, año I, vol. I, enero-febrero 1950, n’ 1 : 59-70.

Rowe, J. H. (1954). El movimiento nacional inca del siglo XVIII. Revista Universitaria. Cuzco, XLIII (107), 17-47.

Stastny, F. (1982). Iconografía, pensamiento y sociedad en el Cuzco virreinal, Cielo Abierto VII, $\mathrm{n}^{\circ}$ 21, Lima.

Tito Cusi Yupanqui, D. D. de ([1570] 1917). Relación de la conquista del Perú y hechos del Inca Manco II, in Libros y documentos referentes a la historia del Peru, t. II.

Valdez, A. ([1782 ?]1938). Ollantay. Ad fidem Codicis Pastor-Justinianiensis. Archivii Nationalis Limensis recensuit. Latine vertit. Integra Codicis Sahuaraurensis lectione. Analysi morphologica grammatica indicibus dissertationibus scholiis auxit Prof. Dr. Hippolytus Galante : 13-44 (fac-similé du manuscrit Justiniani) , 73-149 (transcription et traduction latine de l'acte I) ; Lima : Publicaciones del Instituto Superior de linguistica y filología de la Universidad Mayor de San Marcos. Monumenta Linguae Incaicae.

Vitulli, J. M. (2011). Amar su propia muerte. Madrid: Iberoamericana/Vervuert y Consejo Superior de Investigaciones Científicas.

\section{NOTES}

1. Les Incas appelaient leur empire Tawantinsuyu, littéralement « les quatre bandes ensemble». Ces quatre «bandes» ou parties étaient l'Antisuyu, le Qullasuyu, le Kuntisuyu et le Chinchaysuyu. Toutes recevaient leur nom d'une des principales ethnies qui les habitaient.

2. Une étude approfondie des relations inca-anti se trouve dans Renard-Casevitz et Saignes 1986, pp. 37-129. 
3. Les khipukamayuq ( chargés des khipu»), étaient des fonctionnaires incas qui utilisaient les khipu (khipu «nœud») ou systèmes de cordelettes à nœuds, pour enregistrer des données numériques de nature différente.

4. Il s'agit d'un topos colonial très répandu, hérité des anciens Grecs pour lesquels les Barbares étaient ceux dont la langue se réduisait à des sons incompréhensibles, «bar, bar... ». Voir à ce propos l'article de Géraldine Méret dans ce même dossier.

5. D'après la division verticale du Pérou en huit milieux écologiques, mise au point par le géographe Javier Pulgar Vidal, la yunka correspond à l'étage en-dessous des 2800-2500 m d'altitude. Elle se divise en yunka sèche du versant Pacifique et yunka humide du versant oriental des Andes.

6. Une version du mythe des Hintil se trouve in Itier 2004, pp. 20-39.

7. La plupart des drames du théâtre quechua colonial ont été publiés seulement en traduction espagnole. Voir Meneses 1983.

8. Mes remerciements sincères au département de la recherche et de l'enseignement du Musée du quai Branly et au GDRI pour leur soutien financier qui a rendu possible ma mission auprès du Museo de América de Madrid. Je tiens également à remercier la directrice du Museo de América, María Concepcíon García Saiz, et ses collaborateurs pour la générosité avec laquelle ils ont mis à ma disposition leur collection de queros coloniaux.

9. J'emprunte l'expression comedias marianas à Carlos García-Bedoya, qui désigne ainsi El pobre más rico et l'Uska Pawqar car ces drames sont liés à des sanctuaires mariaux et traitent le thème du salut par l'intercession de la Vierge (García-Bedoya 1998, p. 333).

10. La chronologie du théâtre quechua est de César Itier. À l'exception de El robo de Proserpina, dont les manuscrits et le texte en translittération moderne ont été publiés (Itier, 2010), et d' ollantay, dont j'ai fait moi-même la translittération à partir du manuscrit Justiniani, les autres textes du théâtre quechua sur lesquels se base cette étude, m’ont généreusement été fournis par César Itier en version translittérée. C'est moi qui ai traduit en français les extraits rapportés dans l'article.

11. La maskapaycha était une frange de laine rouge portée sur le front et suspendue au llawt'u, une tresse multicolore qui ceignait la tête de l'Inca.

12. Ayant demandé à l'Inca la main de sa fille et ayant essuyé un refus, Ollantay se retire à Ollantaytambo où il se fait proclamer Inca par les Antis soumis à son autorité.

13. El robo de Proserpina, par exemple, commence par un très long monologue de Pluton-Lucifer, dans lequel le roi des Enfers raconte au public sa révolte contre Dieu, sa défaite et son expulsion du Ciel.

14. Cette laque, appelée verniz de Pasto, est encore aujourd'hui extraite d'une plante, la Mopa-Mopa (Elaeagia pastoensis), qui pousse en Colombie. Il est possible que l'utilisation de la Mopa-Mopa ait

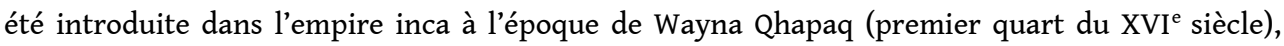
dont les conquêtes inclurent la région de Pastos (Cummins, 2004, p. 187).

15. Les études les plus complètes sur l'iconographie des queros coloniaux se trouvent dans Liebscher, 1986 ; Flores Ochoa et al., 1998 ; Cummins, 2004.

16. Les Incas prêtaient aux Antis des pouvoirs de sorciers et d'herboristes (la plupart des plantes utilisées dans les cérémonies religieuses et dans les rituels de guérison andins, venaient de la forêt). Selon les chroniqueurs, de nombreux prêtres et princes incas auraient fait chez les Antis leur initiation chamanique (Renard-Casevitz et Saignes, 1986, p. 43).

17. Les tuqapu étaient des formes géométriques abstraites insérées dans des cadres carrés ou rectangulaires qui ornaient les tuniques de l'élite inca. Ils indiquaient le statut ethnique, social et religieux de la personne qui les arborait.

18. Dans les portraits des princesses incas réalisés au XVII ${ }^{\mathrm{e}}$ et au XVIII ${ }^{\mathrm{e}}$ siècle par des peintres indigènes, on remarque la présence constante d'un bossu soutenant un parasol de plumes. Les 
sources d'inspiration sont vraisemblablement les peintures baroques espagnoles dans lesquelles figurent des serviteurs nains ou bossus.

19. Francisco de Toledo, vice-roi du Pérou de 1569 à 1581, mena une active campagne de dénigrement contre les Incas afin de priver leurs descendants de toute légitimité de souveraineté sur le pays.

20. Pour une analyse détaillée des instruments de musique représentés dans cette scène, voir Gudemos (2004, pp. 69-70).

21. L'adjectif qhapaq signifie « riche, puissant».

22. Légende recueillie par J. Roel Pineda (1950, p. 61).

23. D'origine précolombienne, la mit'a («tour») était une corvée de travail, repartie par roulement entre les villages tributaires, à laquelle étaient astreints tous les hommes adultes.

\section{ABSTRACTS}

The dweller of the peruvian selva, named Anti or ch'unchu in colonial chronicles, is the protagonist of several figurative scenes painted on the wooden vases called queros. He appears as a shadow also behind the Devil character, a recurring figure of quechua theatre in the Cuzco region.

Comparing examples from these two types of sources (texts and images), chronological contemporaries (XVII ${ }^{\text {th }}$ and XVIII ${ }^{\text {th }}$ centuries) and belonging to the same geographical and cultural area (the Andean highlands), this article propose a new interpretation of the traditional image of the Savage. The perspective moves from the West to the Andes to analyze the way that colonial society looks at this representation of the Other, seen as a counter-mirror through which it seeks to define its own identity. Our vision of the colonial universe will thus be enriched with a new light emphasizing, once again, its complexity.

L'habitant de la selva péruvienne, nommé Anti ou ch'unchu dans les chroniques coloniales, est le protagoniste de plusieurs scènes représentées sur les vases en bois appelés queros. Il apparaît également en filigrane derrière le personnage du Diable, figure récurrente du théâtre en langue quechua de la région de Cuzco. À travers la mise en parallèle d'exemples tirés de ces deux ensembles de documents (textes et images), chronologiquement contemporains (XVII et XVIII ${ }^{\mathrm{e}}$ siècles) et appartenant à la même aire géographique et culturelle (les hautes terres andines), cet article propose une nouvelle interprétation de l'image traditionnelle du Sauvage. La perspective se déplace de l'Occident vers les Andes, pour analyser le regard que la société coloniale, et plus particulièrement l'élite indigène urbaine, porte sur cette représentation de l'Autre, perçu comme un contre-miroir à travers lequel elle cherche à définir sa propre identité. Notre vision de l'univers colonial s'enrichit ainsi d'un nouvel éclairage qui en souligne, une fois de plus, toute la complexité.

El habitante de la selva peruana, llamado Anti o Ch'unchu en las crónicas coloniales, es el protagonista de varias escenas grabadas en vasos de madera denominados queros. Aparece también bajo el personaje del diablo, figura recurrente del teatro en lengua quechua de la región de Cuzco. El análisis paralelo de estas imágenes y de estos textos que fueron creados en una misma región y época (Tierras altas de los Andes, siglos XVII y XVIII) nos lleva a proponer una nueva interpretación de la imagen tradicional del salvaje. Ya no desde occidente sino que desde 
los Andes. A través de la representación del Otro, la élite urbana indígena de la sociedad colonial buscaba definirse a sí misma. Así, se revela tanto la complejidad de la sociedad colonial como de las élites indígenas andinas.

INDEX

Keywords: Antis, ch'unchu, quechua theatre, colonial queros, andean elite

Palabras claves: Antis, ch'unchu, théâtre quechua, queros coloniaux, élite andine

\section{AUTHOR}

\section{ROSSELLA MARTIN}

Institut National de Langues et Civilisations Orientales, France Correo electrónico: rossella.martin@wanadoo.fr 